\title{
Chemosensation and genetic individuality
}

\author{
Prim B. Singh \\ Nuclear Reprogramming Laboratory, Roslin Institute (Edinburgh), Midlothian, \\ Scotland EH25 9PS, UK
}

\begin{abstract}
Numerous studies have shown that there are measurable behavioural consequences that can result from the olfactory recognition of alleles borne at the major histocompatibility complex (MHC). These consequences include simple individual recognition, disassortative mate preference, discrimination of kin from non-kin and whether a pregnancy is carried to term. Such a system, which can influence the reproductive behaviour of a species, will have profound effects on its genetic constitution and survival. The likely mechanism responsible for the production of MHC-related odours involves soluble MHC molecules that carry allele-specific odoriferous molecules from the blood via the kidneys into the urine, from where they are released into the environment. The ability of soluble MHC molecules to signal genetic individuality in this way may have evolved before the appearance of an acquired immune system in our immediate ancestors, the protochordates.
\end{abstract}

Olfactory cues play a fundamental role in social interactions in a wide variety of mammals ranging from rodents to primates (Brown, 1979). Each mammal has an individual odour or signature and individual recognition allows discrimination between members of distinct subgroups and social categories, including the ability to distinguish between kin and non-kin (Porter et al., 1985). Olfactory signatures also have both a releasing and a priming function in mammalian reproduction, and recognition of individuality is influential in each of these functions (MacDonald and Brown, 1985). The chemical nature of olfactory signatures affords several distinct advantages over other sensory cues that can be used for individual recognition. Chemical signals can be deposited on external substrates and can mark out a region or territory even when the individual leaving the mark is no longer present. Unlike visual cues, olfactory signatures are not affected by the rhythm of night and day and can still operate in darkness. Similarly, auditory cues can be perceived only when an animal is awake, whereas olfactory signatures can be recognized independently of the state of arousal (Porter, 1999).

This review is concerned with the olfactory signatures that can reflect the genotype of an individual and thereby provide a relatively stable lifelong label of identity. To this end, studies on the role of the major histocompatibility complex (MHC) in individual recognition are particularly salient. Any discussion of the MHC must draw on many decades of work on its associated immunobiology. This approach is necessary to provide the appropriate background for the behavioural studies.

Email: prim.singh@bbsrc.ac.uk

\section{Immunobiology of classical MHC class I antigens}

First, some basic genetic points. The MHCs of mice and rats are called $\mathrm{H}-2$ and $R T 1$, respectively, and they comprise many linked genes that can be divided into several regions (Fig. 1). The specific loci are called $H-2 K$ and $H-2 D / L$ in mice (Klein, 1979) and they encode the classical MHC class I antigens. The homologous loci in rats are less well defined but have been named RT1-A and RT1-U (Leong et al., 1999; Walter and Gunther, 2000). The particular allele at a given classical class I locus is conventionally denoted by a superscript letter or letters, such as $H-2 K^{b}$ or $R T 1-A^{a}$. Many decades of study have revealed that, in each species, there is a vast number of alleles at the classical MHC class I loci. Although the full extent of the polymorphism is not clearly defined, it has been estimated that, in mice, there are up to 100 alleles at the $K$ and $D$ loci, whereas the $L$ locus is less polymorphic. Similar allele frequencies are estimated for homologous loci in other mammals (Klein, 1986). Thus, in outbreeding mammals, the degree of polymorphism ensures that in each species there are $>10^{9}$ unique class I antigenic phenotypes segregating in the population. The function of forced polymorphism on this scale is not understood.

Classical MHC class I antigens are glycoproteins inserted integrally into the limiting membrane of nearly all cells (Fig. 1). They are best known from studies on tissue transplantation because incompatibility at the MHC causes extreme rejection of grafts. The polymorphism of the MHC therefore represents a barrier to crossmatching of tissues from unrelated donors for organ grafting and also contributes to the variability at the cell surface and to what Medawar (1996) called 'the uniqueness of the individual'. By the same token, only close relatives share similarity at the MHC and thus an individual requiring transplantation must look 


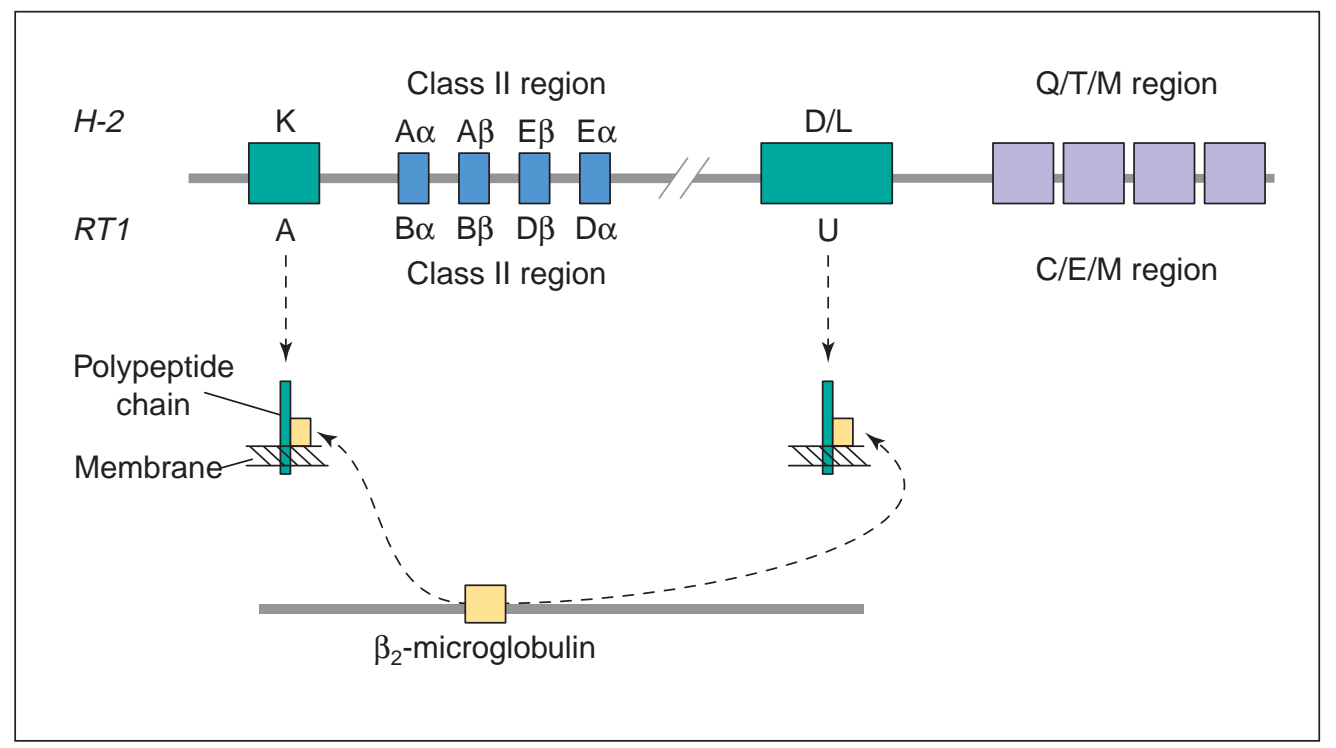

Fig. 1. A schematic illustration of classical class I major histocompatibility complex (MHC) antigens encoded by the mouse $(H-2)$ and rat (RT1) MHCs. The mouse MHC (above the line) is called $\mathrm{H}$-2 and the rat MHC (below the line) is called $R T 1$. The loci encoding the classical highly polymorphic class I antigens are denoted $\mathrm{H}-2 \mathrm{~K}$ and $\mathrm{H}-2 \mathrm{D} / \mathrm{L}$ (green boxes). These loci encode $45 \mathrm{kDa}$ polypeptides that are inserted into the limiting membrane (hatched) of nearly all cells. The polypeptides are non-covalently associated with $\beta 2$-microglobulin $\left(\beta_{2} \mathrm{~m}\right)$, which is encoded by a gene elsewhere in the genome. Also depicted are two other MHC loci that are known to contribute to MHC-associated odours, the class II region and the $\mathrm{Q} / \mathrm{T} / \mathrm{M}$ region. The class III region is not depicted. For a full description of the mouse MHC see Klein (1986). Also shown are the orthologous loci from the rat MHC, RT1. The RT1-A locus corresponds to $\mathrm{H}-2 \mathrm{~K}$ and is made up of three separate loci termed $A_{1}, A_{2}$ and $A_{3}$ (Walter and Gunther, 2000). The RT1-U locus is orthologous to the H-2D/L loci (Leong et al., 1999). Also shown are loci encoding the rat class II polypeptides and the RT1C/E/M region, which is orthologous to the mouse $\mathrm{H}$ $2 \mathrm{Q} / \mathrm{T} / \mathrm{M}$ region. Like those in mice, the classical class I loci in rats encode $45 \mathrm{kDa}$ membrane-bound polypeptides that are non-covalently associated with $\beta_{2}$-microglobulin. The class III loci are not depicted. For a more detailed description of the rat MHC, see Walter and Gunther (2000) and Leong et al. (1999).

to family members to obtain closely matched tissue. Therefore, knowledge of the alleles an individual harbours at the MHC can be used as an indicator of relatedness.

Classical MHC class I antigens consist of two chains: (i) a heavy $(\alpha)$ chain (encoded by the $H-2 K$ and $H-2 D / L$ loci and analogous loci in mammals), of about $45 \mathrm{kDa}$; and (ii) a light $(\beta)$ chain of $12 \mathrm{kDa}$, which is called $\beta_{2}$-microglobulin (Fig. 1). A single copy gene elsewhere in the genome encodes $\beta_{2}$-microglobulin (Kvist and Peterson, 1978). The $\alpha$ and $\beta$ chains associate non-covalently at the plasma membrane and together they are involved in a crucial process during the induction of immune responses, namely in the presentation of foreign antigens to $\mathrm{T}$ lymphocytes (Zinkernagel and Doherty, 1979).

Elegant studies on the atomic structure of class I molecules have revealed how these molecules present antigen to the immune system. A consensus ribbon diagram based on X-ray co-ordinates of a class I molecule is presented (Fig. 2a) (Bjorkman et al., 1987) together with a schematic diagram which clarifies the structural features that are responsible for presentation of antigen to the immune system (Fig. 2b). Key structural determinants are the $\alpha 1$ and $\alpha 2$ domains of the $\alpha$ heavy chain, each of which consists of an $\alpha$ helix and a $\beta$ pleated sheet (Fig. 2b). The two $\beta$ sheets join together to make a platform, which supports the two $\alpha$ helices and thereby defines the so-called antigen-binding cleft. Importantly, the allelic differences (as manifest by amino acid changes) that specify the $\mathrm{MHC}$ antigenic type are concentrated within the floor and walls of this cleft. Thus the polymorphism of the MHC is reflected by the generation of many different types of binding cleft with unique patterns of hydrophobic or charged residues. The binding clefts are normally occupied by self-peptides that are generated by proteolytic degradation of endogenous polypeptides. The spectrum of peptides present in an individual is unique. The peptides enter the cleft during synthesis of the $\alpha$ chain and are present in the cleft before association of $\beta_{2}$-microglobulin with the $\alpha 3$ domain (Fig. 2c). Association of the $\beta$ chain distorts the $\alpha$ chain and locks the peptides in place between the $\alpha$ helices and thereby 


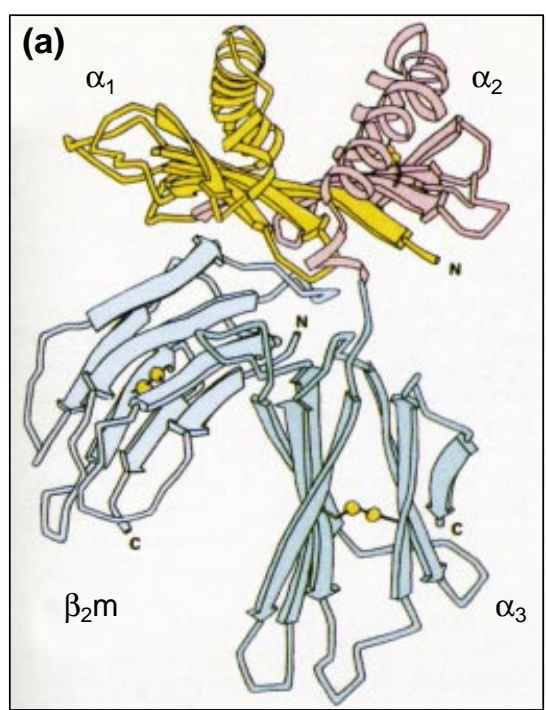

(c)

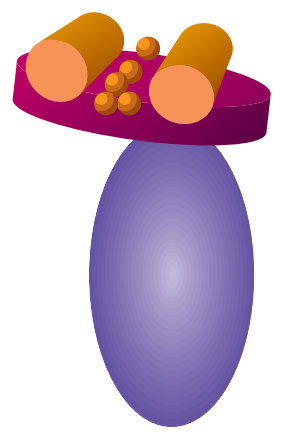

(b)

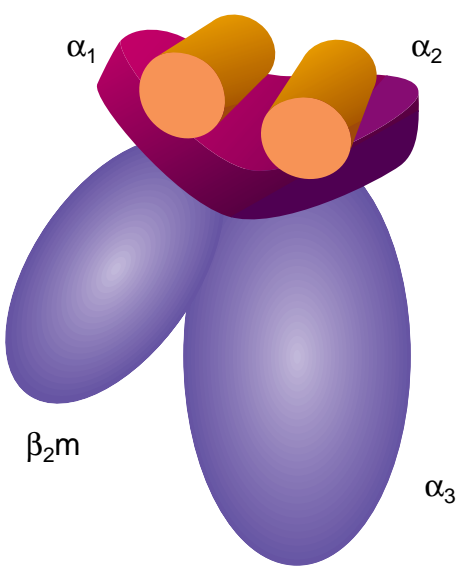

(e)

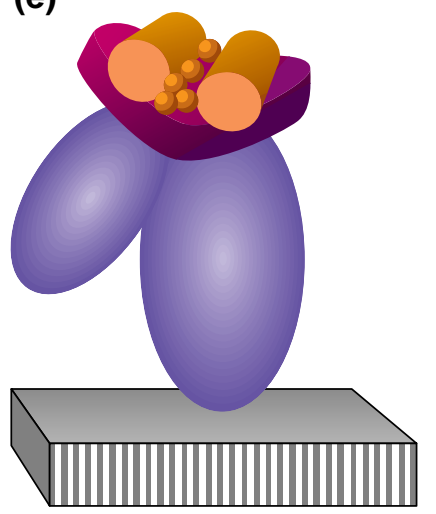

Fig. 2. The atomic structure of $\alpha$ and $\beta$ chains of a classical class I major histocompatibility complex (MHC) molecule and a cartoon depicting the major steps in the assembly of a class I molecule with its associated peptides. $(a, b)$ The heavy chain consists of three domains $(\alpha 1, \alpha 2$ and $\alpha 3)$. The light chain consists of a single domain, $\beta_{2}$-microglobulin $\left(\beta_{2} \mathrm{~m}\right)$, attached non-covalently to the $\alpha 3$ domain. The $\alpha 1$ and $\alpha 2$ domains both consist of an $\alpha$ helix and a $\beta$ pleated sheet. The two sheets join together to make a continuous platform, which supports the two $\alpha$ helices defining an antigen binding cleft (Garrett et al., 1989). (c) Newly synthesized heavy chain consists of a platform of $\beta$ pleated sheet carrying two $\alpha$ helices defining a cleft. The cleft is occupied by a self-peptide of about nine amino acids. The $\alpha 1$ and $\alpha 2$ domains are supported by a globular $\alpha 3$ domain. (d) After peptide binding, the $\alpha 3$ domain associates with the $\beta_{2}$ m light chain via a patch of binding residues. (e) Binding of $\beta_{2}$ m induces a conformational change which enhances the stability of binding of the peptides and readies the molecule for transport to the membrane, where it is anchored by hydrophobic interaction between a transmembrane region and the phospholipid bilayer (hatched rectangle).

ensures the retention of the peptide within the cleft (Kvist and Hamann, 1990; Fig. 2d,e). During infection, exogenous foreign peptides derived from the pathogen are incorporated into the cleft and it is towards this novel complex of $\mathrm{MHC}$-foreign peptide that the immune response is raised.

The demonstration that classical class I molecules are used by $T$ lymphocytes of the immune system as associative recognition molecules has directed attention toward immunological explanations for their polymorphism. The prevalent explanation is that MHC polymorphism in a population ensures that lethal pathogens such as viruses cannot extinguish a species by epidemic infection (Bodmer, 1972). In the absence of polymorphism, mutation of virus proteins to mimic those of the host would result in the presentation of essentially self-peptides to the immune 

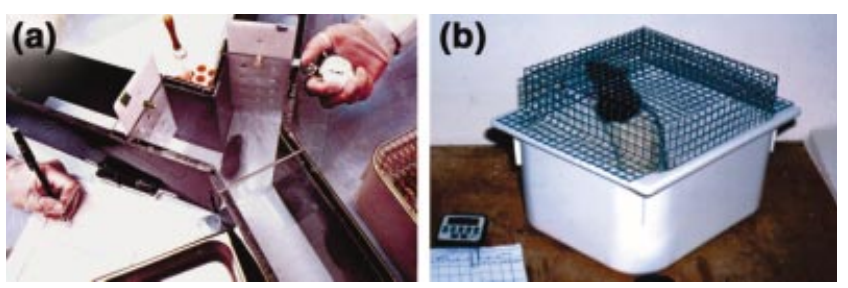

Fig. 3. The $Y$-maze and the habituation-dishabituation assay. (a) Air is drawn through each arm of the Y-maze and the apparatus becomes scented by the odour source, which can be a whole animal or a urine sample. A mouse that has previously been deprived of water is allowed into the 'stalk' of the Y-maze and chooses to enter one of the arms. In the figure, the mouse chooses the right arm. When the choice accords with the odour that has been assigned for reward, a fence is raised allowing the mouse access to a drop of water. Typically, a mouse takes 2 or $3 \mathrm{~s}$ to make its choice either without a pause or with a brief retracing. Placement of the odour source in either the left or right arm is decided by a series of random numbers. For details see Beauchamp et al. (1985). (b) A rat is placed in a test arena and presented with an odour source on a piece of filter paper attached to the lid. If the rat is habituated to the odour it does not respond. If the rat perceives the odour as novel it dishabituates and responds by rearing up on its hind legs and sniffing the air and moving towards the odour source, as depicted in the figure. The time the animal spends investigating the source in this manner is noted.

system, to which an individual is tolerant and towards which an immune response will not be mounted. Such mimicry would ensure that the virus remained free from immune attack. Thus, highly mutable infectious agents would purge the population of common MHC alleles. Through many rounds of virus mutation and selection of rarer MHC types that offer resistance to infection (frequency-dependent selection) the MHC polymorphism would be generated.

There is strong evidence that MHC allele frequencies can be driven by pathogens. The best example is the classical MHC class I allele HLA-Bw53 which appears to confer resistance to malaria in humans (Hill et al., 1991). Allele $B w 53$ is $55 \%$ higher in uninfected individuals than infants infected with severe malaria. In Northern Europe, where malaria is not endemic, the incidence of the $B w 53$ allele is very low, whereas about $40 \%$ of a Nigerian sample was either homo- or heterozygous for this allele. Although frequency-dependent selection explains the occurrence of MHC polymorphism in a species, it does not account for a system that can render nearly every individual in a population antigenically unique. Work on the ability of mice and rats to distinguish between MHC types by olfaction and on the use of this ability to choose between mates, as well as carry a consequent pregnancy to term, has provided another mechanism for maintaining the variation at the MHC (Potts and Wakeland, 1993). This work, which includes our own, is the focus of the remaining sections in this review.

\section{MHC-associated odours and individual recognition}

The discovery that MHC-associated odour signals are present in mice came from the use of genetically defined inbred strains (Yamazaki et al., 1976). Inbred strains consist of animals that are genetically uniform and homozygous for all their genes, that is, each chromosome pair is identical. Two strains that are identical except for allelic differences at a particular locus are called congenic strains, and any measurable characteristic that differs between the two congenic strains must be ascribed to the genes at that locus. Congenic strains of mice and rats that differ only in alleles at the $\mathrm{MHC}$ have been used in several different experimental paradigms to examine whether the MHC is associated with specific odour signatures. The most informative experimental approach is the Y-maze paradigm (Fig. 3a) developed by Yamazaki et al. (1979), which has been crucial in defining the general features of MHC-associated odours and in measuring the olfactory acuity of mice.

\section{The $Y$-maze paradigm}

The basic set-up of the Y-maze is presented (Fig. 3a). Mice are trained to enter alternative chambers scented by an airflow through odour boxes occupied, in the original experiments, by MHC-congenic mice. Mice were trained by water deprivation and reward. In this scheme, a trainee mouse is deprived of water before the experiment (for about $24 \mathrm{~h}$ ) and then given a drop of water when it enters one of the arms of the Y-maze. The mouse first learns to discriminate the differences between two strong odours, normally juniper and cinnamon: it is rewarded, for example, when it enters the cinnamon arm. It is deemed to have learnt the ability to discriminate between the two odours when it chooses the correct arm at least $80 \%$ of the time (degree of concordance). The tasks then become more difficult and the mice are gradually trained to discriminate between two inbred strains and then to distinguish congenic strains that differ only in the alleles at the MHC. In an important development, a generalized (transfer of training) procedure was adopted to ensure that no artefact of the test procedure or unknown variation in odour constitution was responsible for the discrimination (Yamaguchi et al., 1981). Accordingly, previously unused samples of the same odour sources were tested without reward. If the test mice respond correctly to these new samples (concordant response to the learned scent) it can be concluded that incidental or unrelated cues are not involved in the discrimination, it is down to the MHC alone.

Use of the Y-maze paradigm showed that:

- No sensory perception other than olfaction was required to distinguish between MHC types (Yamazaki et al., 1979).

- Urine was as good a source of MHC-specific odours as the whole animal (Yamaguchi et al., 1981).

- Both males and females had equal ability to distinguish between MHC types (Yamaguchi et al., 1981). 
- Olfactory acuity of mice could enable the discrimination of MHC class I molecules that differ only in three amino acids. This was a key observation because it confirmed that MHC-related odours map directly to the class I genes rather than to a related locus (Yamazaki et al., 1983a). Moreover, no amount of training could enable a mouse to distinguish between individuals of the same inbred strain (Yamaguchi et al., 1981).

- Mice could also discriminate between allelic differences in the class II and Qa:Tla (now known as the $\mathrm{Q} / \mathrm{T} / \mathrm{M}$ region; Fig. 1) regions, indicating that all three genetic loci of the MHC can contribute to individual odours (Yamazaki et al., 1982, 1984).

- Use of radiation chimaeras showed that the haemopoietic system is a source of MHC-associated odours (Yamazaki et al., 1985).

- The ease of training indicated that the MHC is as potent a source of individual odours as the rest of the genome taken together (Boyse et al., 1987). $X$ and $Y$ chromosomes can also be discriminated between, but are much less potent in scent marking than the MHC (Yamazaki et al., 1986a).

The Y-maze paradigm enabled researchers to investigate the ability of trained mice to discriminate between MHCrelated odours in the absence of any other variables (stimuli). In contrast, the recognition of MHC-related odours in nature is likely to be both spontaneous and in the context of a variety of other (competing) stimuli. In a possibly more natural paradigm, in which the 'habituation-dishabituation' test was used, it could be shown that rodents can indeed discriminate between MHC types without prior training. The 'habituation-dishabituation' test is a simple and powerful technique for determining whether the subject can detect a difference between two odours (Sundberg et al., 1982).

\section{The 'habituation-dishabituation' paradigm}

This paradigm is based on the simple premise that most mammals are like us: they are usually inquisitive when presented with a novel stimulus, but the novelty can wear off upon extended exposure to the stimulus. The test is very simple and involves placing an experimental animal in a test arena consisting of a plastic box with a removable wire lid. The animal is left in the test arena for a short time so that it has an opportunity to explore and become familiar with it. It is then presented with the first urine odour on a piece of filter paper which is attached to the wire lid. This odour represents a novel stimulus and almost invariably the subject animal responds by orienting to the odour, rearing on its hind legs and sniffing it (Fig. 3b). The animal is presented with the first urine sample three times. By the third exposure, it no longer rears to investigate - it has habituated to the odour. The key test comes in the next trial. When the lid is next changed, a novel urine sample is presented to the animal subject. If it perceives the odour of this new sample to be different from the first sample there is a rapid dishabituation. The rat lifts its head, sniffs the air, and moves toward the new odour and rears to investigate it (Fig. 3b). If the second urine odour is not perceived as different, the animal remains habituated. This experimental paradigm has shown that rats can discriminate between urine samples taken from recombinant congenic inbred strains that differ only in the alleles at the RT1-A classical class I locus (Singh et al., 1987). Further work has shown that the class II region of the MHC can also contribute to the production of individual odours (Brown et al., 1989). Thus, the olfactory acuity of rats is similar to that of mice and they have been used in this experimental paradigm to investigate the mechanisms involved in the elaboration of MHCassociated odours.

\section{MHC-associated odours and reproduction}

Spontaneous recognition of the MHC was first observed in the original experiments on mating preference, in which a male mouse was presented with congenic females that differ at the MHC (Yamazaki et al., 1976). The male, who is the recipient of the alternative MHC signals, is thought to have the active role and usually, though not always, chooses a female that is different from him at the MHC. A more recent experiment indicates that females can also have an active role in choosing mates. A study on seminatural populations of mice, which nest communally and can mate freely, showed that females choose mates and prefer to mate with males of different MHC (Potts et al., 1991). This observation is in agreement with theoretical considerations which predict that it is more important for females than males to choose mates since their genetic investment is greater (Trivers, 1972): after formation of the zygote the female has the sole responsibility for development of the embryo and care of the young.

The effect of the MHC on murine reproduction is by no means limited to mate preference. Experiments designed around the well-known 'Bruce effect' showed that recognition of the MHC could have clear neuroendocrine effects that influenced the ability of mothers to carry a pregnancy to term. The Bruce effect is the abortion of pregnancy during the preimplantation stage of development brought about by exposure of the pregnant female to a strange male (Bruce, 1959). This effect can be seen when the only differences between the stud and strange males are in the alleles of the MHC (Yamazaki et al., 1983b). Indeed, it was shown that abortion could be elicited when the only genetic differences between the stud and the foreign male were three point mutations in the $\mathrm{H}-2 \mathrm{~K}^{\mathrm{b}}$ molecule $\left(\mathrm{H}-2 \mathrm{~K}^{b}\right.$ for the stud versus $\mathrm{H}-2 \mathrm{~K}^{\mathrm{bm} 1}$ for the strange male and vice versa; Yamazaki et al., 1986b). Abortion can be prevented by administration of prolactin or progesterone (Dominic, 1966a), indicating that the effect is likely to be due to neuroendorine disturbance. Moreover, it was demonstrated that the urine of the foreign male contains the pheromone that elicits the effect, since bedding or urine from the foreign 


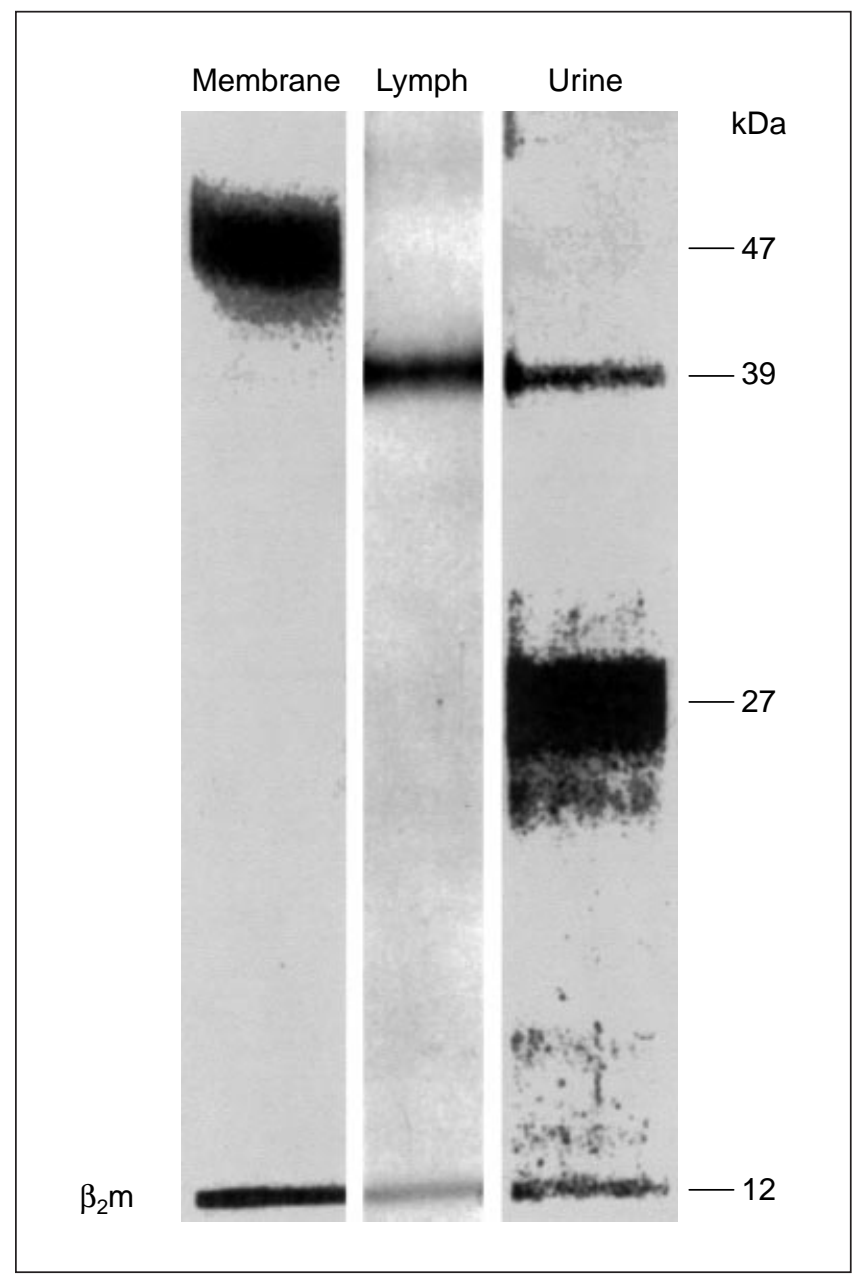

Fig. 4. The different forms of rat major histocompatibility complex (MHC) class I molecules purified from the membrane, the circulation and the urine. Membrane: when class I molecules are detergent-solubilized from cell membranes, purified and separated on a polyacrylamide gel, the heavy chain $(\alpha)$ has a molecular mass of $47 \mathrm{kDa}$. This molecule consists of the entire $\alpha$ chain $(\alpha 1, \alpha 2$ and $\alpha 3$ domains, the transmembrane region and cytoplasmic tail). It is associated with $\beta_{2}$-microglobulin $\left(\beta_{2} \mathrm{~m}\right)$, which runs at the bottom of the gel and has a molecular mass of $12 \mathrm{kDa}$. Lymph: in the circulation, the heavy chain is reduced to $39 \mathrm{kDa}$. This smaller molecule only has the $\alpha 1, \alpha 2$ and $\alpha 3$ domains and lacks the transmembrane region and cytoplasmic tail. It remains associated with equimolar quantities of $\beta_{2} \mathrm{~m}$. This soluble molecule is equivalent to the molecule that can be released from membranes by a papain digestion (Pearse-Pratt et al., 1999). Urine: the major band in the urine runs at $27 \mathrm{kDa}$ and represents a fragment equivalent to the $\alpha 1$ and $\alpha 2$ domains of the heavy chain. A similar fragment can be produced after extensive digestion of purified class I molecules with papain, which can cleave class I molecules at a site between the $\alpha 2$ and $\alpha 3$ domains (Pearse-Pratt et al., 1999). The $\alpha 1-\alpha 2$ fragment does not associate with $\beta_{2} \mathrm{~m}$ and any $\beta_{2} \mathrm{~m}$ seen in this lane is due to that associated with the $39 \mathrm{kDa}$ molecules that can be found in urine. male is as good a source of the odours as the whole animal (Parkes and Bruce, 1962; Dominic, 1966b).

MHC-based mating preferences and pregnancy blocking may provide additional selective pressures that act to maintain $\mathrm{MHC}$ polymorphism. This will be discussed later when the evolutionary implications of $\mathrm{MHC}$ recognition are considered. The following section is concerned with mechanisms that might underpin the elaboration of $\mathrm{MHC}$ associated odours.

\section{MHC-associated odours: the 'carrier hypothesis'}

Three mechanisms have been proposed to explain how the MHC might produce an odour of individuality. First, on the basis of evidence that MHC polymorphism can determine variation in the development of organs and cell populations, it has been suggested that MHC-based developmental variations give rise to distinctive odour profiles (Boyse et al., 1987). This hypothesis is difficult to test and it will not be discussed further. A second hypothesis was based upon the known functions of the MHC as encoding molecules involved in the restrictive recognition of foreign antigen (Howard, 1977). It was argued that the intimate linkage of MHC class I genotype with urinary odour was indirect and reflected the immune response against commensal bacterial flora of the skin, urinary tract and gut. Individual MHC types would be associated with unique flora. The volatile odorants in the excretions were thought to be secondary metabolites derived from these organisms. A priori this hypothesis seemed unconvincing since it requires that the types and relative numbers of commensal bacteria are constant over time, which is known not to be the case (Savage, 1977). More importantly, three experiments using the $\mathrm{Y}$-maze paradigm have shown that immune regulation of commensal flora is not necessary for the determination of MHC-associated odours. Firstly, it is possible to discriminate between urine samples taken from germ-free $\mathrm{MHC}$ congenic mice, which lack commensal flora (Yamazaki et al., 1990). Secondly, radiation chimaeras reconstituted with $\mathrm{F}_{1}$ bone marrow smell like the $\mathrm{MHC}$ of the bone marrow donors, rather than the recipients (Yamazaki et al., 1985). Thus, the MHC-associated odours in these chimeras are not determined by $\mathrm{MHC}$ restriction of the immune response, which is heavily skewed towards that of the recipient (Katz et al., 1978), but reflects the genotype of the bone marrow donor. This finding is consistent with the idea that the genotype of haematopoietic/lymphoid cells is the crucial determinant of MHC-related odours (Yamazaki et al., 1985; Singh et al., 1988). Finally, it has been observed that fetal MHC-associated odours are evident in the urine of mothers as early as day 9 of gestation (Beauchamp et al., 1994), before the fetus has a functioning immune system (Owen and Ritter, 1969). Clearly, neither bacterial flora nor immune responses are necessary for the elaboration of $\mathrm{MHC}$ associated odours.

We have presented a third mechanism for the elaboration of MHC-associated odours, the 'carrier hypothesis' (Singh, 
(a)

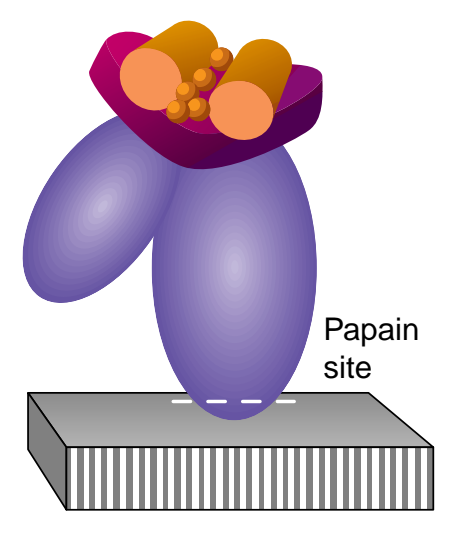

(b)

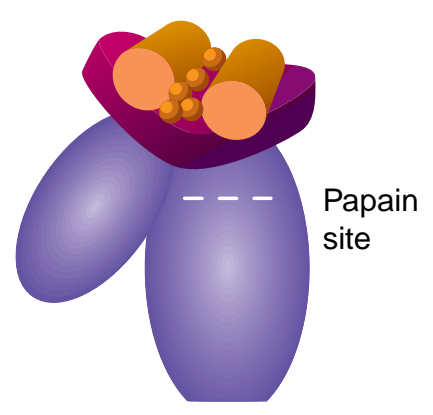

(c)
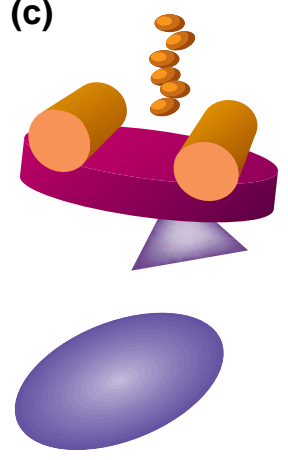

(d)

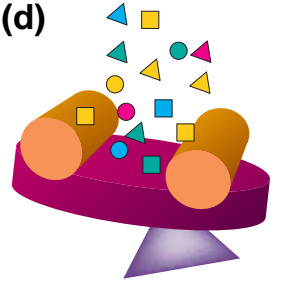

Fig. 5. A cartoon depicting a hypothetical route of class I major histocompatibility complex molecules from the membrane to the circulation and into the urine. (a) Cleavage of the molecule at the juxta-membranous protease site removes the transmembrane region and releases the molecule into the circulation in soluble form. (b) The molecule does not aggregate and circulates as a soluble heterodimer consisting of the $\alpha$ and $\beta$ chains. (c) Further enzymatic cleavage at the junction of the $\alpha 2$ and $\alpha 3$ domains removes most of the $\alpha 3$ domain with the attached $\beta_{2}$-microglobulin. This cleavage allows relaxation of the binding platform, opening of the cleft and loss of bound peptide. (d) The resulting molecule with a naked binding cleft is capable of binding a cocktail of small aromatic molecules such as volatile odorants and is small enough to pass into the urine.

1986, 1999; Singh et al., 1987), which can accommodate all the evidence to date. The idea for this hypothesis came from work in rats which showed that classical MHC class I antigens are not only found as membrane-bound molecules but also in true solution, in the lymph, blood and urine (Singh, 1986; Singh et al., 1987). The serum molecule is a heterodimer with a heavy chain of $39 \mathrm{kDa}$ associated noncovalently with $\beta_{2}$-microglobulin (Fig. 4). Proven sources of the molecules are macrophages and dendritic cells of haematopoetic/lymphoid lineage (Singh et al., 1988). They are present in the serum at a concentration between 350 and $390 \mathrm{ng} \mathrm{ml}^{-1}$, have a short half-life of $2.7 \mathrm{~h}$ and are excreted into the environment via the kidneys (Singh et al., 1988). In the urine, the molecules undergo degradation, giving rise to a major molecular mass species of $27 \mathrm{kDa}$ (Fig. 4), corresponding to cleavage at the known papain cleavage site at the junction between the $\alpha 2$ and $\alpha 3$ domains of the heavy chain (Fig. 5c). Thus, classical class I molecules, which in their membrane-bound form act as markers of tissue individuality, are excreted in the urine and might serve as individuality markers in the environment, which can be detected by olfaction (Singh, 1986).

Class I molecules are unlikely candidates for the odoriferous components in the urine as they are large and consequently lack a vapour pressure. However, drawing on their role as associative molecules in the immune system, it was suggested that class I molecules might associate with smaller molecules, in an allele-specific way, and transport them from the blood into the urine (Singh, 1986; Singh et al., 1987, 1988). Thus a unique mixture of volatiles would be selected from a common pool of metabolites, to which commensal flora could contribute (Singh et al., 1990), and would be transported to the urine to impart a unique MHCspecific odour. The advantages of such carrier proteins as mediators of olfactory signals are well known (Albone, 1984): the slow steady release of small volatiles from the carrier; control of excretion of the volatile molecules; and protection of these volatiles from decomposition.

The proposed hypothesis was tested by Pearse-Pratt et al. (1999). Purified class I molecules of the RT1-A a type were injected into normal PVG.RT1 ${ }^{\mathrm{u}}$ animals which possess the $R T 1^{u}$ haplotype. The effect of this procedure was measured in a habituation-dishabituation assay and it was shown that the smell of the urine taken from the injected animals had been affected. Specifically, the urine samples taken from the animals was found to be indistinguishable from urine taken from PVG.R8 animals, which belong to a recombinant inbred strain that possesses the $A^{a}$ allele at the RT1-A locus and are otherwise identical to the PVG.RT1 ${ }^{\mathrm{u}}$ animals. The clear explanation for this result is that injection of the RT1$\mathrm{A}^{\mathrm{a}}$ molecule transferred the authentic $R T 1-A^{a}$ odour to the PVG.RT1u urine. This explanation is entirely consistent with the 'carrier hypothesis'.

Given these data, it is possible to sketch a molecular mechanism by which class I molecules pick up unique mixtures of volatile odorants via their binding cleft and transport them into the urine to be used in individuality marking (Fig. 5). Accordingly, soluble class I molecules are generated by proteolytic cleavage from cells at a juxtamembraneous cleavage site (Fig. 5a). The soluble molecules with their associated peptides are thereby released into the circulation (Fig. 5b). They undergo further fragmentation by 


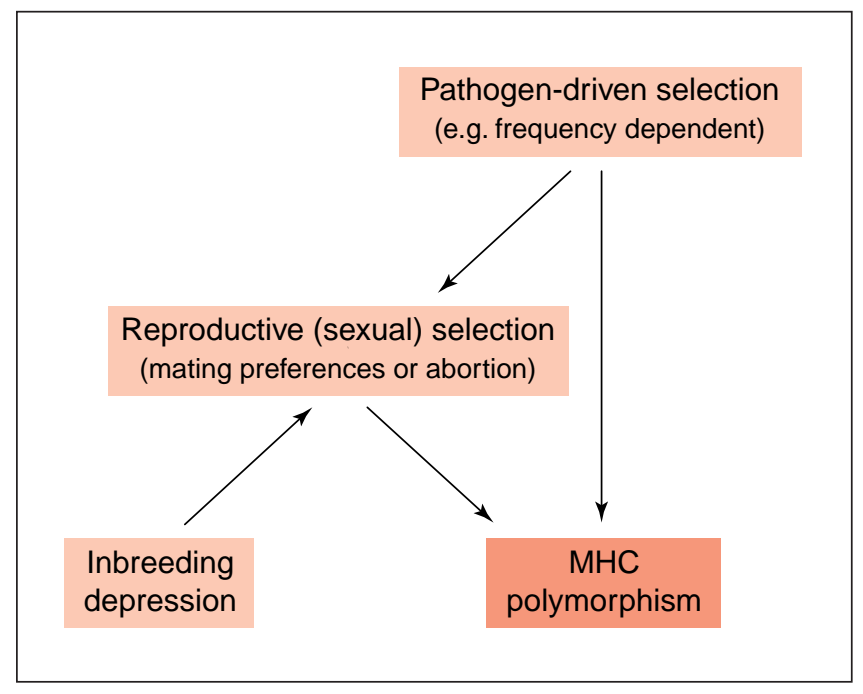

Fig. 6. The selective pressures that drive major histocompatibility complex (MHC) polymorphism in mice. The direction of the arrows indicates the selective forces that favour MHC polymorphism. These include the two types of pathogen-driven selection, namely frequency-dependent selection and heterozygous advantage (over-dominance) (Bodmer, 1972). More indirectly, disassortative mating preferences based on the MHC will also increase MHC polymorphism. It is thought that premating mechanisms of this type are more efficient at generating polymorphism that those involving pregnancy blocking, since premating mechanisms do not involve the costs associated with mating and aborted embryos. For further details see Potts and Wakeland (1993).

proteases in the body fluids, giving rise to the major proteolytic $27 \mathrm{kDa}$ fragment in the urine, which represents cleavage of the binding cleft (the joined $\alpha 2$ and $\alpha 3$ domains) away from the rest of the molecule (Fig. 5c). The effect of this degradative pathway is relaxation of the binding platform, opening of the cleft and release of any bound peptides (Fig. 5c). The now empty platform can bind a unique cocktail of odorants (Fig. 5d), which is transported into the urine where further degradation leads to release of the molecules and the production of an MHC-specific odour. There is good evidence that proteolysis is necessary for production of MHC-associated odours (Yamazaki et al., 1999). Serum has an odour but cannot be used to discriminate between MHC types (Brown et al., 1987; Yamazaki et al., 1999). However, when sera from MHCcongenic mice are treated with proteases, MHC-specific odorants are released and discrimination between MHC types is possible (Yamazaki et al., 1999). This finding indicates that the MHC-specific odourants are present prerenally in serum and that their liberation requires proteolysis. The nature of the specific odorant molecules that are bound to soluble class I molecules is unknown, although carboxylic acids may be one source (Singer et al., 1997).

\section{MHC-associated odours: evolutionary considerations}

It has been argued that the unprecedented diversity of MHC classical class I genes is due to pathogen-driven selection that favours rare MHC alleles (Bodmer, 1972). Under both laboratory conditions (Yamazaki et al., 1976) and in semiwild populations (Potts et al., 1991) mice prefer to mate with individuals that are different from them at the MHC. The combination of these two selective pressures, pathogen-driven and reproductive selection, allowed Potts and Wakeland (1993) to propose a simple model to explain the evolution of MHC polymorphism (Fig. 6). Potts and Wakeland (1993) proposed that the generation of MHC polymorphism is primarily driven by pathogens (for example frequency-dependent selection). This selection in turn allows the evolution of MHC-dependent disassortative mating preferences because these matings produce progeny that are heterozygous at the $\mathrm{MHC}$ and thus possess a greater fitness (for example they have better defence against highly mutable pathogens). Pregnancy blocking (the Bruce effect) can also be incorporated into this scheme; as discussed earlier, an established pregnancy can be aborted if a female is presented with the opportunity to mate with a male that is different at the MHC, which may function to avoid inbreeding (Yamazaki et al., 1983b). Another effect of increasing MHC polymorphism by pathogen-driven and reproductive selection is that it also increases the usefulness of the MHC as a marker of genetic relationships (Potts and Wakeland, 1993). This is because the more polymorphic the MHC in a population, the less likely non-kin will share alleles at this locus. This feature has long been recognized in medicine in the fact that the best donors for tissue grafting are kin of the recipient.

Much theoretical work has been done on the evolution of altruism and how kin recognition mechanisms might be used to enhance the inclusive fitness of genes (Hamilton, 1964; Dawkins, 1976). The MHC is particularly salient to this work because its polymorphism enables a measure of the genome-wide relatedness: only related individuals are likely to share alleles at the MHC. Its role in kin recognition was addressed in a series of experiments in which it was shown that female mice usually choose nest mates that are similar at the MHC (Manning et al., 1992). These studies were conducted using semi-wild populations in which the animals had freedom of choice with regard to mating and nesting. Under these conditions, female mice nested communally, gave birth at about the same time and suckled each other's pups indiscriminately. Unexpectedly, the choice of nest mate appeared to be made on the basis of the MHC: nest mates normally shared alleles at the MHC. Moreover, in an important control using congenic strains of mice, it was shown that the choice of kin could not be explained by shared alleles at other genetic loci. Thus recognition of kin was synonymous with recognition of the alleles at the MHC and it was similarity at the MHC that mattered.

The use of MHC or MHC-like antigens as a system to 

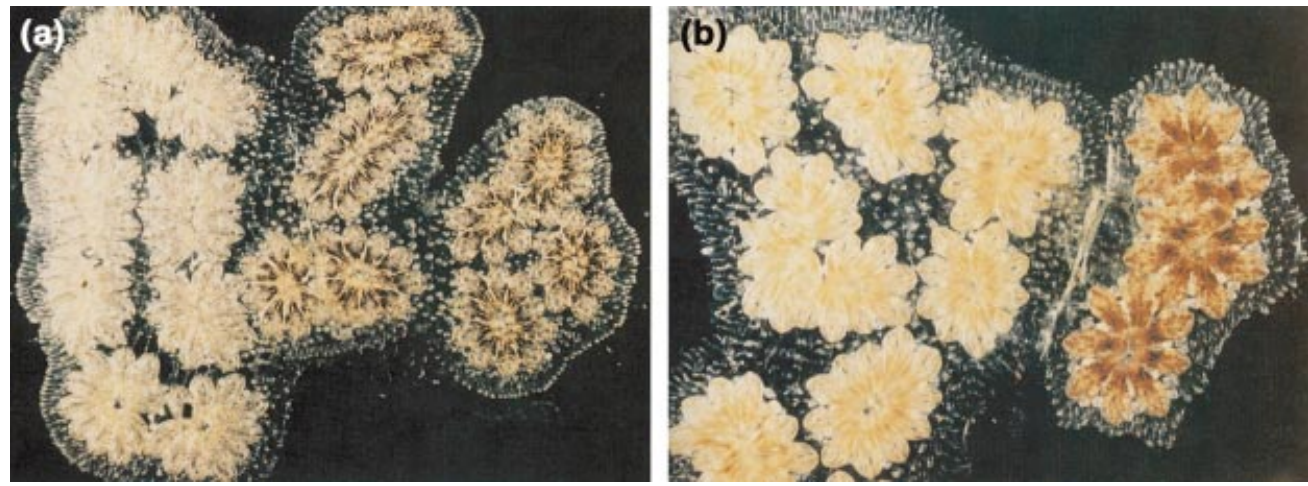

Fig. 7. Fusion and rejection in paired colonies of Botryllus schlosseri. (a) Two colonies that share histocompatibility alleles are placed together. Viewed from above, the two vasculature systems become interconnected and gradually become indistinguishable from each other, although initially the two colonies retain their respective pigment patterns. (b) Two colonies that differ at the histocompatibility loci are placed together. There is an inflammatory process associated with rejection whereby the tunic surrounding the colonies becomes opaque, which is seen as a thickened line between the colonies. For details see Magor et al. (1999).

discriminate kin from non-kin is likely to extend beyond vertebrates. Unlike the situation in vertebrates, natural tissue rejection is well documented in our immediate ancestors, the protochordates (Burnet, 1971; Magor et al., 1999). More importantly, rejection is due to the recognition of highly polymorphic histocompatibility antigens (Scofield et al., 1982), which can also be used to discriminate between kin and non-kin (Grosberg and Quinn, 1986). In the colonial tunicate, Botyllus schlosseri, two colonies that share alleles at the histocompatibility loci are able to fuse together, whereas disparity leads to necrosis at the interface between colonies, rejection and often the death of the smaller colony (Fig. 7). It is not known whether the histocompatibility loci encode true structural homologues of their vertebrate counterparts. (Whether modern-day MHC molecules are structurally related to the histocompatibility antigens found in protochordates is not germane to the argument. For an individuality system based on histocompatibility antigens to work, the antigens should be polymorphic and it should be possible for the members of the species to detect this variation.) Nevertheless, there is evidence that soluble forms of these histocompatibility antigens might be involved in a recognition system that enables discrimination between histocompatibility types. Larvae of B. schlosseri live as free-swimming plankton, which eventually settle and form a colony. Genotyping nearest neighbours of settled larvae has shown that there is a significant preference to settle close to larvae that share identity at the histocompatibility loci (Grosberg and Quinn, 1986). This recognition does not involve direct physical contact and thus requires a vector by which the larvae can recognize each other's histocompatibility type. The most likely candidates for the vector are soluble forms of the histocompatibility antigens. This example has been considered a primitive form of kin recognition in which relatedness is measured solely by recognition of histocompatibility alleles. It may also represent the ultimate in altruistic behaviour because the larvae that settle close to each other and share histocompatibility alleles have greater opportunity to fuse and form a single colony. By sharing resources in this way, a bigger (fitter) colony is formed.

\section{MHC-associated odours: conclusions}

It is well known that polymorphism at the MHC enables this genetic locus to act as a tissue individuality marker. Less well known is the fact that there are measurable behavioural consequences when mice and rats perceive this genetic individuality by olfaction. In the appropriate context, recognition of the MHC can lead to simple individual recognition, disassortative mate preference, discrimination of kin from non-kin and can determine whether a pregnancy is carried to term. Thus, as originally envisaged by Thomas (1974), MHC-based recognition systems extend beyond immune surveillance to influencing the behaviour of animals. There is evidence that humans produce an MHC-specific odour (Ferstl et al., 1990; Eggert et al., 1999), which correlates with the presence of soluble MHC molecules (Wobst et al., 1999). The olfactory acuity in humans also extends to the ability to discriminate between MHC types (Gilbert et al., 1986). However, it is unclear whether, in the welter of other social and biological factors, the MHC influences human behaviours such as mating preference and maintenance of pregnancy (see Wedekind et al., 1995; Beauchamp and Yamazaki, 1997; Penn and Potts, 1998). This area requires further study.

Our work on the mechanisms by which MHC-associated odours might be produced indicates that MHC molecules 
could act as vectors that transport volatile metabolites into the environment. These volatiles, which are peculiar to each MHC type, represent the odoriferous component that is recognized by smell. The evolution of individual recognition, based on $\mathrm{MHC}$-like loci, is likely to pre-date the appearance of an acquired immune system because protochordates, which have an innate immune system, possess an MHC-like system that can be used to discriminate between individuals. Such evidence indicates that individual recognition in many animals could involve soluble highly polymorphic histocompatibility-like antigens.

The author would like to thank Peter Sharp for his interest in this work and the opportunity to write this review. He would also like to thank Geoffrey Butcher for information on the rat MHC and Norrie Russell and lan Cowell for help with artwork.

\section{References}

Key references are identified by asterisks.

Albone AS (1984) Mammalian Semiochemistry John Wiley and Sons, New York

Beauchamp GK and Yamazaki K (1997) HLA and mate selection in humans: commentary American Journal of Human Genetics 61 494-496

Beauchamp GK, Yamazaki K and Boyse EA (1985) The chemosensory recognition of genetic individuality Scientific American 253 86-92

Beauchamp GK, Yamazaki K, Curran M, Bard J and Boyse EA (1994) Fetal $\mathrm{H}-2$ odortypes are evident in the urine of pregnant female mice Immunogenetics 39 109-113

Bjorkman PJ, Saper MA, Samraoui B, Bennett WS, Strominger JL and Wiley DC (1987) The foreign antigen binding site and T cell recognition regions of class I histocompatibility antigens Nature 329 512-518

Bodmer WF (1972) Evolutionary significance of the HL-A system Nature 237 139-145

Boyse EA, Beauchamp GK and Yamazaki K (1987) The genetics of body scent Trends in Genetics 3 97-102

Brown RE (1979) Mammalian social odors: a critical review Advances in the Study of Behavior 10 103-162

Brown RE, Singh PB and Roser B (1987) The major histocompatibility complex and the chemosensory recognition of individuality in rats Physiology and Behavior 40 65-73

Brown RE, Roser B and Singh PB (1989) Class I and class II regions of the major histocompatibility complex both contribute to individual odors in congenic inbred strains of rats Behavioural Genetics 19 659-674

Bruce HM (1959) An exteroceptive block to pregnancy in the mouse Nature 184 105-106

Burnet MF (1971) "Self-recognition" in colonial marine forms and flowering plants in relation to the evolution of immunity Nature 232 230-235

Dawkins R (1976) The Selfish Gene Oxford University Press, New York

Dominic CJ (1966a) Observations on the reproductive pheromones of mice. II. Neuro-endocrine mechanisms involved in the olfactory block to pregnancy Journal of Reproduction and Fertility 11 415-421

Dominic CJ (1966b) Observations on the reproductive pheromones of mice. I. Source Journal of Reproduction and Fertility 11 407-414

Eggert F, Luszyk D, Haberkorn K, Wobst B, Vostrowsky O, Westphal E, Bestmann HJ, Muller-Ruchholtz W and Ferstl R (1999) The major histocompatibility complex and the chemosensory signalling of individuality in humans Genetica 104 265-273

Garrett TP, Saper MA, Bjorkman PJ, Strominger JL and Wiley DC (1989) Specificity pockets for the side chains of peptide antigens in HLA-Aw68. Nature 342 692-696

Gilbert AN, Yamazaki K, Beauchamp GK and Thomas L (1986) Olfactory discrimination of mouse strains (Mus musculus) and major histocompatibility types by humans (Homo sapiens) Journal of Comparative Psychology $100262-265$
*Grosberg RK and Quinn JF (1986) The genetic control and consequences of kin recognition by the larvae of a colonial invertebrate Nature 322 456-458

Hamilton WD (1964) The genetical evolution of social behaviour Journal of Theoretical Biology 7 17-50

Hill AV, Allsopp CE, Kwiatkowski D, Anstey NM, Twumasi P, Rowe PA, Bennett S, Brewster D, McMichael AJ and Greenwood BM (1991) Common west African HLA antigens are associated with protection from severe malaria Nature 352 595-600

Howard JC (1977) H-2 and mating preferences Nature 266 406-408

Katz DH, Skidmore BJ, Katz LR and Bogowitz CA (1978) Adaptive differentiation of murine lymphocytes. I. Both $\mathrm{T}$ and B lymphocytes differentiating in F1 transplanted to parental chimeras manifest preferential cooperative activity for partner lymphocytes derived from the same parental type corresponding to the chimeric host Journal of Experimental Medicine $148727-745$

Klein J (1979) The major histocompatibility complex of the mouse Science 203 516-521

Klein J (1986) The Natural History of the Major Histocompatibility Complex Wiley, New York

Kvist S and Hamann U (1990) A nucleoprotein peptide of influenza A virus stimulates assembly of HLA-B27 class I heavy chains and beta 2microglobulin translated in vitro. Nature 348 446-448

Kvist S and Peterson PA (1978) Isolation and partial characterization of a beta2-microglobulin-containing, $\mathrm{H}-2$ antigen-like murine serum protein Biochemistry 17 4794-4801

Leong LY, Le Rolle AF, Deverson EV et al. (1999) RT1-U: identification of a novel, active, class Ib alloantigen of the rat MHC Journal of Immunology $162743-752$

MacDonald DW and Brown RE (1985) Introduction: the pheromone concept in mammalian chemical communication. In Social Odours in Mammals pp 1-18 Vol. 1 Eds RE Brown and DW MacDonald. Clarendon Press, Oxford

Magor BG, De Tomaso A, Rinkevich B and Weissman IL (1999) Allorecognition in colonial tunicates: protection against predatory cell lineages? Immunological Reviews 167 69-79

Manning CJ, Wakeland EK and Potts WK (1992) Communal nesting patterns in mice implicate $\mathrm{MHC}$ genes in kin recognition Nature 360 581-583

Medawar PB (1996) A biological analysis of individuality Clinical Orthopaedics 326 5-10

Owen JJ and Ritter MA (1969) Tissue interaction in the development of thymus lymphocytes Journal of Experimental Medicine 129 431-442

Parkes AS and Bruce HM (1962) Pregnancy-block in female mice placed in boxes soiled by males Journal of Reproduction and Fertility 4 303-310

Pearse-Pratt R, Schellinck H, Brown R, Singh PB and Roser B (1999) Soluble $\mathrm{MHC}$ antigens and olfactory recognition of genetic individuality: the mechanism Genetica 104 223-230

Penn D and Potts W (1998) How do major histocompatibility complex genes influence odor and mating preferences? Advances in Immunology 69 411-436

Porter RH (1999) Olfaction and human kin recognition Genetica 104 259-263

Porter RH, Cernoch JM and Balogh RD (1985) Odor signatures and kin recognition Physiology and Behavior 34 445-448

Potts WK and Wakeland EK (1993) Evolution of MHC genetic diversity: a tale of incest, pestilence and sexual preference Trends in Genetics 9 408-412

*Potts WK, Manning CJ and Wakeland EK (1991) Mating patterns in seminatural populations of mice influenced by MHC genotype Nature 352 619-621

Savage DC (1977) Microbial ecology of the gastrointestinal tract Annual Reviews in Microbiology 31 107-133

Scofield VL, Schlumpberger JM, West LA and Weissman IL (1982) Protochordate allorecognition is controlled by a MHC-like gene system Nature 295 499-502

Singer AG, Beauchamp GK and Yamazaki K. (1997) Volatile signals of the major histocompatibility complex in male mouse urine Proceedings National Academy of Sciences USA 94 2210-2214

Singh PB (1986) Classical Class I MHC Antigens in Solution in the Body Fluids PhD Thesis, Cambridge University 
Singh PB (1999) The present status of the 'carrier hypothesis' for chemosensory recognition of genetic individuality Genetica 104 231-233

*Singh PB, Brown RE and Roser B (1987) MHC antigens in urine as olfactory recognition cues Nature 327 161-164

Singh PB, Brown RE and Roser BJ (1988) Class I transplantation antigens in solution in the body fluids and the urine: individuality signals to the environment Journal of Experimental Medicine 168 195-211

Singh PB, Herbert J, Roser B, Arnott, L, Tucker DK and Brown RE (1990) Rearing rats in a germ-free environment eliminates their odours of individuality Journal of Chemical Ecology 16 1667-1682

Sundberg H, Doving K, Novikov S and Ursin H (1982) A method for studying responses and habituation to odors in rats Behaviour Neural Biology 34 113-119

Thomas L (1974) Fear of Pheromones: The Lives of a Cell pp 16-19 Viking, New York

Trivers RL (1972) Parental investment and sexual selection. In Sexual Selection and the Descent of Man pp 56-87 Ed. B Campbell. Chicago University Press, Chicago

Walter L and Gunther E (2000) Physical mapping and evolution of the centromeric class I gene-containing region of the rat $\mathrm{MHC}$ Immunogenetics $\mathbf{5 1}$ 829-837

Wedekind C, Seebeck T, Bettens F and Paepke AJ (1995) MHC-dependent mate preferences in humans Proceedings of the Royal Society of London Series B Biological Sciences $260245-249$

Wobst B, Zavazava N, Luszyk D, Lange K, Ussat S, Eggert F, Ferstl R and Muller-Ruchholtz W (1999) Molecular forms of soluble HLA in body fluids: potential determinants of body odor cues Genetica 104 275-283

Yamaguchi M, Yamazaki K, Beauchamp GK, Bard J, Thomas L and Boyse EA (1981) Distinctive urinary odors governed by the major histocompatibility locus of the mouse Proceedings National Academy of Sciences USA 78 5817-5820

Yamazaki K, Boyse EA, Mike V, Thaler HT, Mathieson BJ, Abbott J, Boyse J, Zayas ZA and Thomas L (1976) Control of mating preferences in mice by genes in the major histocompatibility complex Journal of Experimental Medicine 144 1324-1335

*Yamazaki K, Yamaguchi M, Baranoski L, Bard J, Boyse EA and Thomas L (1979) Recognition among mice. Evidence from the use of a Y-maze differentially scented by congenic mice of different major histocompatibility types Journal of Experimental Medicine 150 755-760

Yamazaki K, Beauchamp GK, Bard J, Thomas L and Boyse EA (1982) Chemosensory recognition of phenotypes determined by the Tla and $\mathrm{H}$ $2 \mathrm{~K}$ regions of chromosome 17 of the mouse Proceedings National Academy of Sciences USA 79 7828-7831

Yamazaki K, Beauchamp GK, Egorov IK, Bard J and Boyse EA (1983a) Sensory distinction between $\mathrm{H}-2^{b}$ and $\mathrm{H}-2^{b m 1}$ mutant mice Proceedings National Academy of Sciences USA 80 5685-5688

Yamazaki K, Beauchamp GK, Wysocki CJ, Bard J, Thomas L and Boyse EA (1983b) Recognition of $\mathrm{H}-2$ types in relation to the blocking of pregnancy in mice Science 221 186-188

Yamazaki K, Beauchamp GK, Thomas L and Boyse EA (1984) Chemosensory identity of $\mathrm{H}-2$ heterozygotes Journal of Molecular and Cellular Immunology $\mathbf{1}$ 79-82

Yamazaki K, Beauchamp GK, Thomas L and Boyse EA (1985) The hematopoietic system is a source of odorants that distinguish major histocompatibility types Journal of Experimental Medicine 162 1377-1380

Yamazaki K, Beauchamp GK, Matsuzaki O, Bard J, Thomas L and Boyse EA (1986a) Participation of the murine $X$ and $Y$ chromosomes in genetically determined chemosensory identity Proceedings National Academy of Sciences USA 83 4438-4440

Yamazaki K, Beauchamp GK, Matsuzaki O, Kupniewski D, Bard J, Thomas $\mathbf{L}$ and Boyse EA (1986b) Influence of a genetic difference confined to mutation of $\mathrm{H}-2 \mathrm{~K}$ on the incidence of pregnancy block in mice Proceedings National Academy of Sciences USA 83 740-741

*Yamazaki K, Beauchamp GK, Imai Y, Bard J, Phelan SP, Thomas L and Boyse EA (1990) Odor types determined by the major histocompatibility complex in germfree mice Proceedings National Academy of Sciences USA 87 8413-8416

Yamazaki K, Beauchamp GK, Singer A, Bard J and Boyse EA (1999) Odortypes: their origin and composition Proceedings National Academy of Sciences USA 96 1522-1525

Zinkernagel RM and Doherty PC (1979) MHC-restricted cytotoxic T cells: studies on the biological role of polymorphic major transplantation antigens determining T-cell restriction-specificity, function, and responsiveness Advances in Immunology 27 51-177 